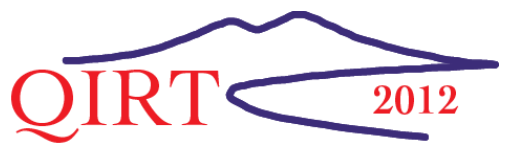

\title{
Gradient-emissivity thermal emitter for IR camera testing and calibration
}

\author{
by H. Polakowski*, T. Piątkowski*, G. De Mey*, B. Więcek ${ }^{* *}$, R. Olbrycht** and K. Firmanty* \\ *Institute of Optoelectronics, Military University of Technology, Warsaw, Poland, hpolakowski@wat.edu.pl \\ ** Institute of Electronics, Technical University of Lodz, Poland, wiecek@p.lodz.pl \\ *** Electronic and Information Systems Dept. University of Gent, Belgium, demey@elis.ugent.be
}

\section{Abstract}

This paper presents design and testing a reference grey body fabricated using different-depth microcavities. The apparent emissivity is achieved by varying the shape coefficient $K$ defined as depth/width ratio. Three different values of shape factor were used in the measurements, $K=1.6,4$, 9. There are 2 versions of the emitter made of aluminium and copper, with different coatings made of titanium and nickel. Beneath the emissive surface, a Peltier cooler/heater is located with a closed loop temperature regulation system. The proposed emitter allows changing the emissivity sharply, and therefore can be applied everywhere the simultaneous measurement of different infrared radiation levels is required. It is dedicated for testing and calibrating modern observations and fully calibrated IR systems. The paper presents the radiation heat transfer analysis of the emitter based on configuration factors. The numerical, analytical and measurement results are compared.

\section{Gradient emissivity IR emitter}

There are black bodies available on the market. Some of them are very expensive, especially if the precise temperature regulation is required. The cheaper solution can be realized just by using the body with modulated emissivity [1-5]. The patent [5] of the emitter was submitted and now the description of the device can be presented in the papers.

The active surface of the emitter was made using Wire Electro Discharge Machining (WEDM) method. This method allows creating the deep grooves as shown in fig. 1a. During metal processing, the uniform high quality isotropic surface can be made. Such surface is close to a perfect diffusive Lambertian plane, however it is not flat - fig. $1 \mathrm{~b}$.

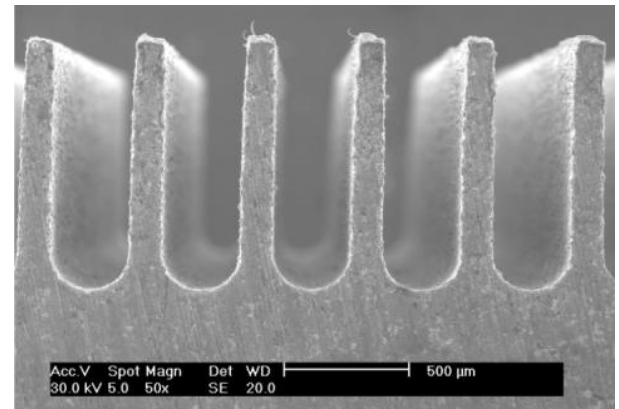

a)

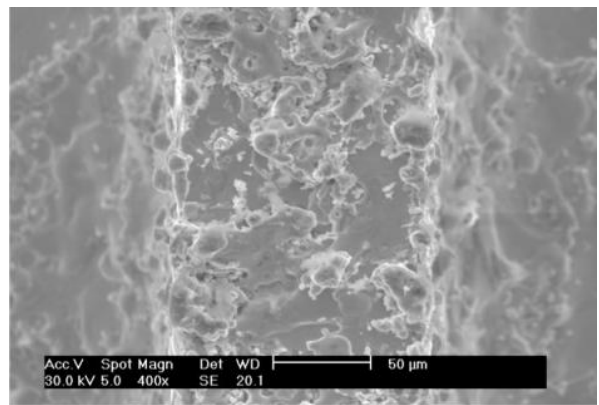

b)

Fig. 1. Scanning microscope photography: a) a comb structure for the gradient-emissivity IR emitter, b) flat surface of IR emitter made with WEDM method

In the copper substrate, 3 patterns of grooves with different shape factors $K$ were made. Factor $K$ is defined as the ratio between the height $h$ and the width of the groove $K=h / a$-fig 4 . In the work we were using the grey body with $K=1.7$, 4 and 9 - fig. 2.

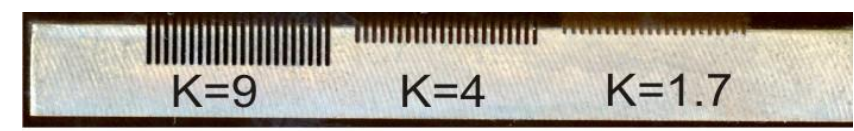

$50 \mathrm{~mm}$

Fig. 2. Profile view of gradient emissivity grey body (IR emitter)

The surface was passivated with the thin layer of metallization using RF sputtering. In order to have more sections with different emissivity coefficients 2 different thicknesses of metallization was made with different metals: nickeltitanium, aluminium-wolfram and gold. Finally, each substrate has sections with 6 different emissivity coefficients, as shown in fig. 3. 


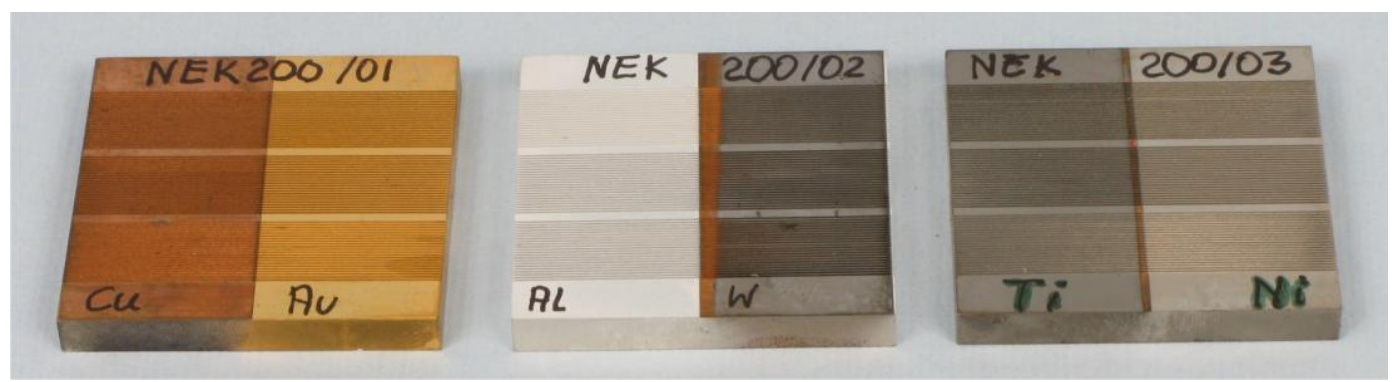

Fig. 3. Gradient emissivity IR emitters

The thermal contrast of the emitter can easily be changed by varying the shape factor $K$. In addition, the radiation intensity depends on the flat material emissivity the emitter in made of. The thermal contrast can be varied by the substrate temperature which is set using the Peltier heater/cooler.

\section{Theoretical analysis}

A series of grooves were made in a material a cross sectional view is shown in fig. 4.

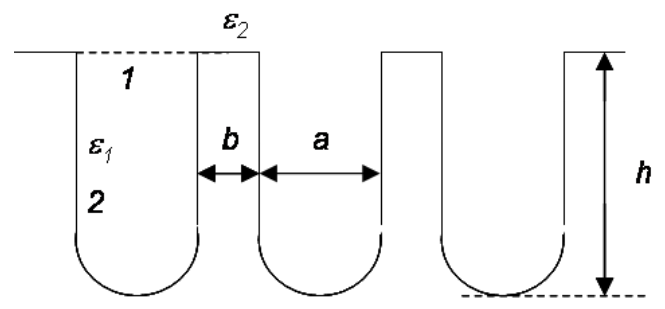

Fig. 4. Cross-section of the groove used for modeling

If we consider just one groove, denoting the top surface by the label 1 and the internal wall by 2 , it is known that the geometry factor $F_{1,2}=1$. Using the relation $S_{1} F_{1,2}=S_{2} F_{2,1}$, whereas $S_{1}$ and $S_{2}$ denote the areas of the surfaces 1 and 2, we obtain eqn. (1).

$$
F_{2,1}=\frac{S_{1}}{S_{2}}=\frac{a}{2 h+\pi \frac{a}{2}}
$$

Using $F_{2,1}+F_{2,2}=1$, which means that all radiation originating from 2 can fall on either 1 or 2 , one gets eqn (2)

$$
F_{2,2}=1-F_{2,1}=1-\frac{a}{2 h+\pi \frac{a}{2}}
$$

If one is looking from above, the surface 1 has an apparent emissivity $\varepsilon_{a p p}$ given by eqn. (3) [7].

$$
\varepsilon_{\text {app }}=\frac{\varepsilon_{2}}{1-\left(1-\varepsilon_{2}\right) F_{2,2}}
$$

where $\varepsilon_{2}$ is the emissivity of the material the vertical walls are made of. Due to the mechanical treatment, the roughness of the flat top layer can be quite different from the internal wall 2, so that different emissivities are generally possible $\varepsilon$ and $\varepsilon_{2}$.

A thermographic camera is looking from above towards the top surface, which is at a uniform temperature but due to the grooves with a spatially varying emissivity. It is also assumed that the camera is at a sufficiently long distance so that every pixel of the detector matrix will receive radiation from the grooves as well as the space between them.

If the ambient is at a temperature $T_{a}$, the amount of radiation coming from the top surface can be expressed by eqn.

$$
\frac{a \varepsilon_{a p p} \sigma T^{4}+b \varepsilon_{1} \sigma T^{4}+a\left(1-\varepsilon_{a p p}\right) \sigma T_{a}{ }^{4}+b\left(1-\varepsilon_{1}\right) \sigma T_{a}{ }^{4}}{a+b}=\sigma T^{4}{ }_{\text {disp }}
$$

If the emissivity correction of the thermographic camera is not active, the camera displays a temperature $T_{\text {disp }}$, which corresponds to an emitted radiation given by $\sigma T^{4}$ disp. In order to make a physical interpretation easier, it is assumed now that all temperatures are close to the ambient temperature $T_{a}$. 


$$
\begin{aligned}
& T=T_{a}+\Delta T \\
& T_{\text {disp }}=T_{a}+\Delta T_{\text {disp }}
\end{aligned}
$$

Linearization of the equation (4) gives the approximation $(T+\Delta T)^{4} \approx T^{4}+4 T^{3} \Delta T$ and it leads to the formula (6).

$$
\Delta T_{\text {disp }}=\frac{a \varepsilon_{a p p}+b \varepsilon_{1}}{a+b} \Delta T
$$

Changing $\varepsilon_{a p p}$ by making the grooves deeper, the camera will observe a different temperature $\Delta T_{\text {disp }}$ even when the object remains at the same temperature $\Delta T$. If one is looking at two regions with different depth of grooves, one will measure a temperature difference given by eqn. (7)

$$
\Delta T_{\text {disp ,1 }}-\Delta T_{\text {disp ,2 }}=\frac{a}{a+b}\left(\varepsilon_{a p p, 1}-\varepsilon_{a p p, 2}\right) \Delta T
$$

If one is looking at three different regions one can finally write eqn. (8).

$$
\frac{\Delta T_{\text {disp , } 1}-\Delta T_{\text {disp , },}}{\Delta T_{\text {disp , }}-\Delta T_{\text {disp }, 3}}=\frac{\varepsilon_{\text {disp , }}-\varepsilon_{\text {disp , }}}{\varepsilon_{\text {disp }, 1}-\varepsilon_{\text {disp }, 3}}
$$

Remark that the last expression does no longer depend on the temperature value. Only material parameters are left. Effective emissivity of the grey body depends on values $a$ and $b$ and can be expressed as in eqn. (9). A plot of the effective emissivity versus shape factor defined as $K=h / a$ is presented in fig. 5 .

$$
\varepsilon=\frac{a \varepsilon_{a p p}+b \varepsilon_{1}}{a+b}
$$

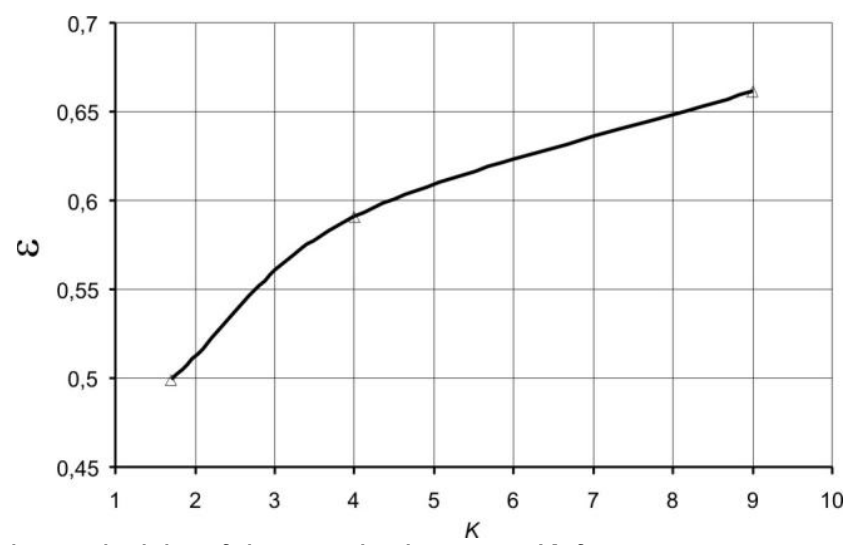

Fig. 5. Effective emissivity of the grey body versus $K$, for $a=250 \mu \mathrm{m}, a=125 \mu \mathrm{m}, \varepsilon_{1}=\varepsilon_{2}=0.25$

\section{Measurement results}

In this work the results of measurement of a grey body with nickel and titanium coating are presented. The IR emitter during testing is presented in fig. 6. The Peltier cooler/heater is applied to stabilize the emitter temperature. A thermocouple ( $\mathrm{K}$ type) is used for monitoring the temperature during the test. In addition, the temperature difference $\Delta T$ between the emitter and the ambient is measured.

In order to observe the contrast inversion, one was tuning the temperature from $38{ }^{\circ} \mathrm{C}$ to $20{ }^{\circ} \mathrm{C}$. It allowed identifying the thermal time constant of the emitter as well - fig. 7 . Thermal time constant is above $100 \mathrm{~s}$. Thermodiagram of the emitter surface for time $t=0$, with the contact measured temperature $T=38{ }^{\circ} \mathrm{C}$ is presented in fig. $8 \mathrm{a}$. Three areas are indicated there for titanium and nickel coatings for shape factor $K=1.7,4$ and 9 . Fig. $8 \mathrm{~b}$ shows the detailed view of the grooves with close up optics.

Finally, we estimated the emissivity for the sections of the IR emitter with $\mathrm{Ti}$ and $\mathrm{Ni}$ layers as a function of $\mathrm{K}$ factor fig. 9 . 

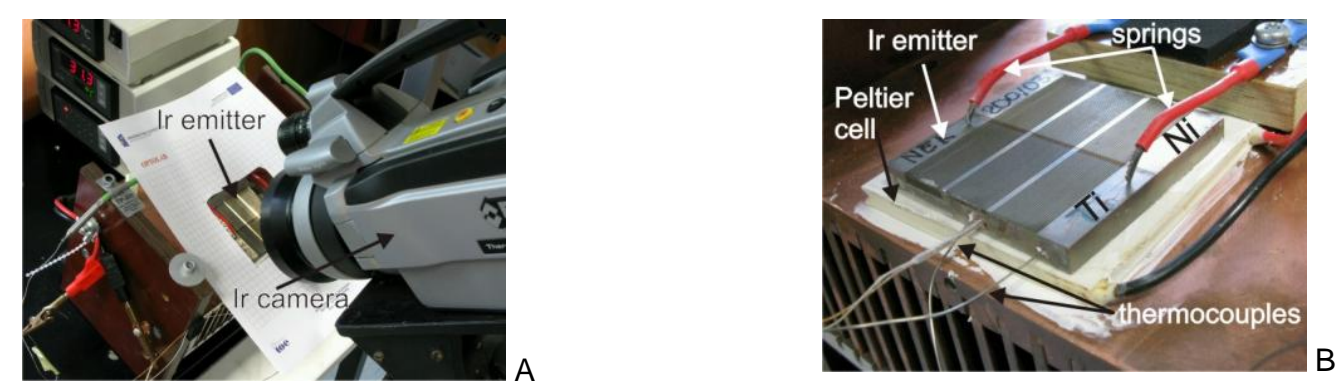

Fig.6. IR emitter testing setup for thermovision measurements

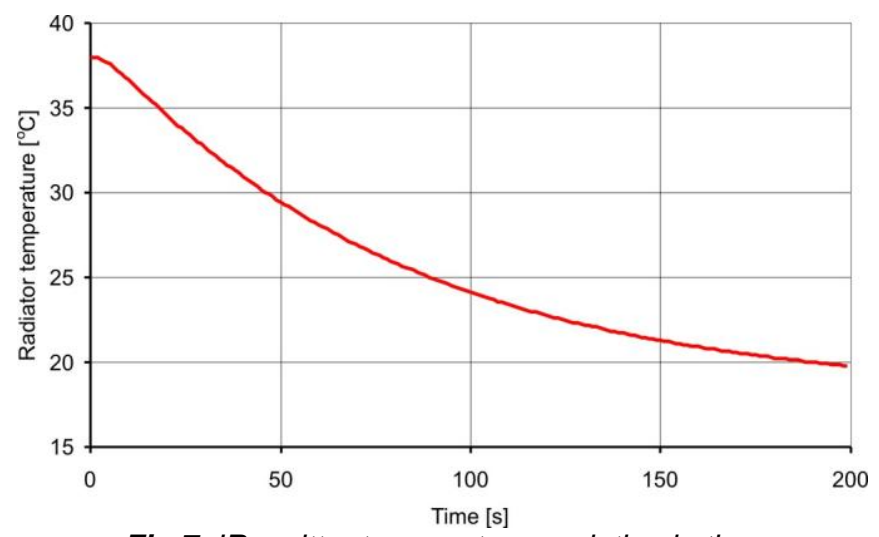

Fig.7. IR emitter temperature evolution in time

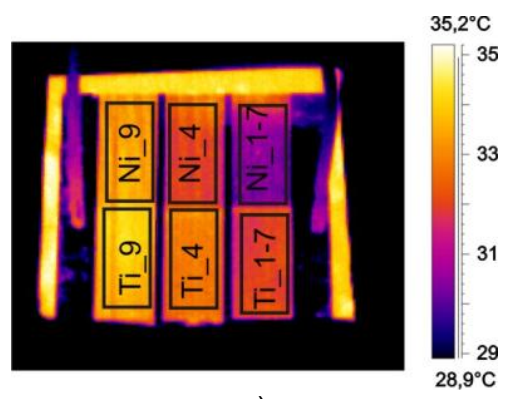

a)

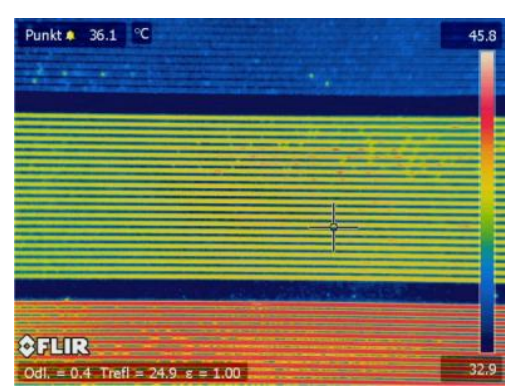

b)

Fig. 8. IR image of gradient emissivity grey body, a) IR emitter@ $38^{\circ} \mathrm{C}$, b) close up IR image, IR emitter@38 ${ }^{\circ} \mathrm{C}$

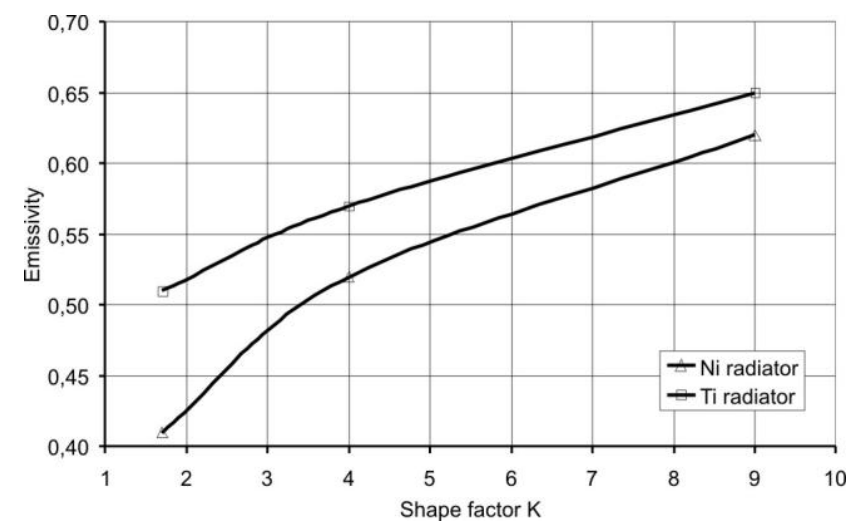

Fig. 9. Effective emissivity vs shape factor $K$ for different section of IR 
A final test was performed using the thermographic camera. The IR emitter radiation temperature was monitored while its temperature was varying from 25 to $37^{\circ} \mathrm{C}$ - fig. 10. The images were recorded with 1 frame/s frame rate. Six regions of interest at the emitter surface were monitored. As it is expected the curves cross out if the emitter temperature reaches the temperature $T_{a} \approx 29.5{ }^{\circ} \mathrm{C}$. Normally this should happen at ambient temperature $\left(25.3^{\circ} \mathrm{C}\right)$. This discrepancy is due to the fact that in this experiment the temperature range was scanned in 200 s so that no steady state conditions were obtained. In addition, the thermocouple used for contact temperature measurement was located $2 \mathrm{~mm}$ below the surface of the emitter.

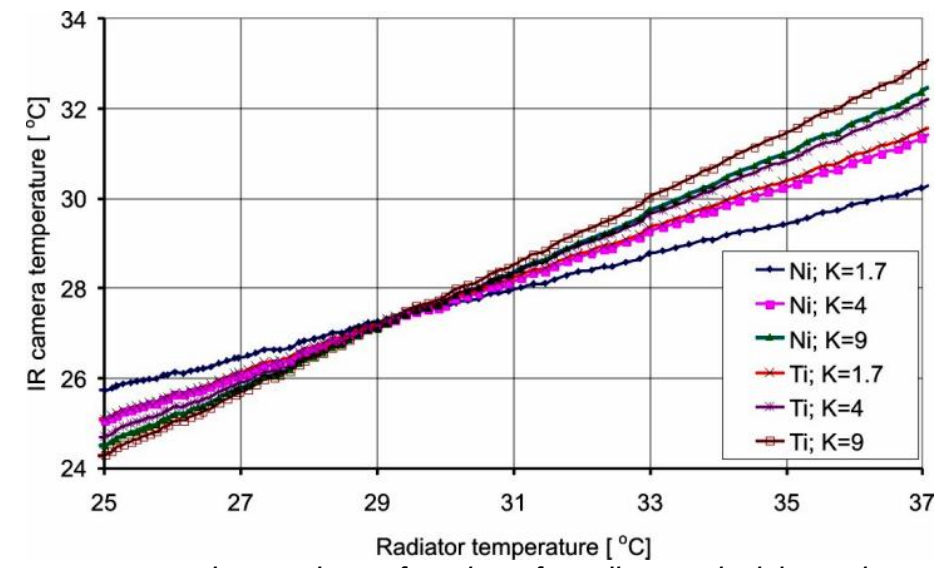

Fig. 10. IR camera temperature observed as a function of gradient emissivity emitter temperature (ambient temperature $25.3^{\circ} \mathrm{C}$ )

When the curves in fig. 10, the inverse of contrast between the pair for given sectors inverses. The contrast inversion temperature in slightly different for each pair of sectors and is varies from $29.05^{\circ} \mathrm{C}$ to $29.73^{\circ} \mathrm{C}$. It is presented in details in table 1.

Table 1. Temperature of IR emitter for contrast inversion

\begin{tabular}{|c|c|c|}
\hline $\begin{array}{c}\text { "Ni" emitter } \\
\text { temperature }\end{array}$ & $\begin{array}{c}\text { "Ti" emitter } \\
\text { temperature }\end{array}$ & $\begin{array}{c}\text { Temperature contrast inversion of the } \\
\text { pair of surfaces }\end{array}$ \\
\hline${ }^{\circ} \mathrm{C}$ & ${ }^{\circ} \mathrm{C}$ & shape factor 1/shape factor 2 \\
\hline 29.27 & 29.05 & $4 / 9$ \\
\hline 29.44 & 29.33 & $1.7 / 9$ \\
\hline 29.60 & 29.73 & $1.7 / 4$ \\
\hline
\end{tabular}

\section{Conclusion}

We have developed a grey body with varying emissivity factors. It was realized by changing the ratio of depth and width of the microcavites. The experimental and theoretical simulation results were compared and they confirmed the possibility of using such device for camera testing and calibration. It is worth to notice that the radiation temperature contrast for the different sections of the emitter can vary with ambient temperature, and can even be inverted.

\section{REFERENCES}

[1] H. Polakowski, N. Hots, T. Piatkowski, T. Orżanowski, Multi-cavity grey body for testing and calibration of pyrometers, Advanced Infrared Technology and Applications AITA 9, Leon, 2007, pp. $249-254$.

[2] T. Piatkowski, H. Polakowski, N. Hots, Examination of metallic surfaces for IR gray body sources, 9th International Conference on Quantitative InfraRed Thermography, Cracow, Poland, 2008, pp 185-190.

[3] H. Polakowski, T. Piątkowski, R. Dulski, K. Firmanty, M. Morawski, Wielosektorowy promiennik podczerwieni, PAK no. 11/2009, pp. 894-897, (in Polish).

[4] H. Polakowski, T. Piątkowski, Testowe promienniki podczerwieni, PAK no. 11/2011, pp. 1351-1354, (in Polish).

[5] H. Polakowski, T. Piątkowski, R. Dulski, P. Trzaskawka, Segmentowy promiennik podczerwieni, patent submission P-392680 (18.10.2010).

[6] Robert Siegel, John R. Howell, Thermal radiation heat transfer, Taylor \& Francis, 2002.

[7] B. Więcek, G. DeMey, Termografia w podczerwieni, PAK, 2011, (in Polish). 